\title{
Effect of hysterectomy on genital infections
}

\author{
FRANKLYN N. JUDSON AND MICHAEL A. RUDER \\ From the Denver Metro Health Clinic, Disease Control Service, Denver Department of Health and \\ Hospitals, Denver, Colorado, USA
}

SUMMARY In a study of women with anogenital gonorrhoea who had undergone hysterectomy, the sensitivities of urethral, vaginal, and anal canal cultures were $88 \cdot 9,55 \cdot 7$, and $40 \cdot 7 \%$ respectively in specimens from 27 women. To obtain the greatest sensitivity, however, we recommend that specimens from all three sites should be cultured routinely. After the raising effects of trichomoniasis and menstrual blood on the $\mathrm{pH}$ values have been allowed for, the vaginal $\mathrm{pH}$ of 74 women in the study group and of 137 healthy controls was similar.

\section{Introduction}

The prevalence and course of sexually transmitted genital infections could theoretically be influenced by the absence of the uterine body and cervix. Several factors may play a part, such as the predilection of gonococci for endocervical columnar epithelium (Harkness, 1948), the pH-buffering effect of cervical mucus (Peeters et al., 1972), the bacteriological effects of mucin (Enhorning et al, 1970), the competition from endocervical bacterial flora and their metabolic products (Bartlett et al., 1978), and the presence of menstrual blood (Cohen, 1969). Our aim was to study how surgical removal of the cervix affects the prevalence of sexually transmitted genital infections by comparing disease-specific infection rates in women who had undergone hysterectomy with rates in a control group of women with an intact uterus. In addition, we have attempted to identify the most sensitive site for culturing Neisseria gonorrhoeae from women after hysterectomy.

\section{Patients and methods}

The Denver Metro Health Clinic is a major clinic for the treatment of sexually transmitted diseases (STD) in the Denver metropolitan area. The clinic records about 22000 patient visits each year and more than $65 \%$ of the cases of gonorrhoea reported from Denver are treated there.

A standardised clinic visit form was used to collect an extensive STD data base for each patient

Address for reprints: Dr F. N. Judson, Disease Control Service, 605 Bannock Street, Denver, Colorado 80204, USA

Received for publication 5 March 1979
(Rothenberg and Judson, 1979). The data base did not include the reason(s) for the hysterectomy or whether or not oophorectomy had also been performed. In this study, women were considered to be 'symptomatic' if they complained of vaginal discharge.

\section{CULTURE TECHNIQUES}

At the inital visit (a first-time or a subsequent visit more than 30 days after a previous visit), two specimens for culture for gonococci were obtained from the endocervix and one from the anal canal (without a proctoscope) of healthy control women whereas one specimen was obtained from each of the urethra, posterior vaginal fornix, and anal canal of women who had undergone hysterectomy. Calciumalginate urethrogenital swabs were used for urethral specimens whereas cotton-tipped swabs were used for specimens from other sites. Specimens were streaked directly on to modified Thayer-Martin medium and placed in an incubator at $36^{\circ} \mathrm{C}$ in an atmosphere containing 5-10\% carbon dioxide. After 40-48 hours' incubation, cultures showing morphologically typical oxidase-positive colonies of Gram-negative diplococci were considered to give a positive result.

Cotton-swab specimens for saline wet preparations and $10 \%$ potassium hydroxide $(\mathrm{KOH})$ mounts were obtained from the posterior vaginal fornix of all women and examined microscopically within five minutes for the presence of trichomonads and yeasts. Vaginal discharge was graded according to (a) quantity-none, scanty (insufficient quantity to flow into the lower half of the speculum), moderate (sufficient quantity to flow into the speculum but not from the introitus), and profuse (spontaneous flow from the introitus); and (b) quality-clear, white, or 
purulent. Finally, the $\mathrm{pH}$ value of the posterior vaginal secretions was determined with nitrazine paper (phenophthiazine paper, E. R. Squibb and Sons Inc., Princeton, New Jersey) in all study group women and in a $50 \%$ sample of the healthy control group.

\section{DEMOGRAPHIC DATA}

\section{Study group}

From 26 May 1977 to 13 May 197891 women under 40 years of age who had undergone hysterectomy presented to the clinic. The age limit was applied to avoid any effects of the menopause on the vaginal $\mathrm{pH}$ value and on the infection rates of the control group of women.

\section{Control group}

Out of 8141 women with an intact uterus seen during the same period a control group of 264 women (three controls for 82 women and two controls for nine women), under 40 years of age was selected by computer for identical composition to the study group for age (mean 32.6 years), race ( $78 \%$ white, $10 \%$ black, $10 \%$ Hispanic, and $2 \%$ others), sexual activity (mean $2 \cdot 1$ different sexual partners within $\mathbf{3 0}$ days of the clinic visit), period since most recent sexual contact (mean 5.8 days), and history of exposure to gonorrhoea $(15 \cdot 4 \%$ had been exposed within 30 days of the clinic visit).

To determine the relative sensitivities of urethral, vaginal, and anal canal cultures for $N$. gonorrhoeae more accurately, we continued to investigate the study group until December 1978.

Differences between the study group and the control group were analysed using a critical ratio for the difference of proportions ( $\mathrm{z}$ test). The differences in $\mathrm{pH}$ were examined with an unpaired Student's $t$ test for comparison of means.

\section{Results}

Vaginal discharge was significantly $(z=3 \cdot 5847$, $\mathrm{P}<0.001)$ more common in the study group $(\mathbf{7 7 \%})$ than in the control group (56\%). This difference was also noted for subgroups with gonorrhoea $(75 \%$ compared with 44\%), trichomoniasis (88\% compared with $76 \%)$ and yeast infection $(90 \%$ compared with $\mathbf{7 4 \%}$ ). In contrast, there was no significant difference between the two groups in terms of quantity and quality of vaginal discharge as determined by the clinician during vaginal examination. Thus, it appears that women who have undergone hysterectomy are more aware, or less tolerant, of vaginal discharge.

Comparative rates of genital infection are shown in Table 1. Trichomoniasis was significantly more common in the study group than in the controls; on the other hand, for gonorrhoea and yeast infection there were no significant differences. In the study group, seven $(50.6 \%)$ of 14 women exposed to gonorrhoea were infected compared with $19(65 \cdot 5 \%)$ of 29 in the control group ( $P=$ not significant).

In Table 2, the range of posterior vaginal $\mathrm{pH}$

Table 2 Comparison of vaginal pH in 74 women who had undergone hysterectomy and in 137 healthy controls, none of whom had trichomoniasis or were menstruating

\begin{tabular}{|c|c|c|c|c|}
\hline \multirow[b]{2}{*}{$p H$ value } & \multicolumn{2}{|c|}{ Study group $(n=74)$} & \multicolumn{2}{|c|}{ Control group $(n=137$} \\
\hline & No. & $\%$ of total & No. & $\%$ of total \\
\hline $\begin{array}{l}4 \cdot 5 \\
5 \cdot 0 \\
5 \cdot 5 \\
6 \cdot 0 \\
6 \cdot 5 \\
7 \cdot 0 \\
7 \cdot 5\end{array}$ & $\begin{array}{r}34 \\
14 \\
11 \\
8 \\
5 \\
2 \\
0\end{array}$ & $\begin{array}{c}45 \cdot 9 \\
18 \cdot 9 \\
14 \cdot 9 \\
10 \cdot 8 \\
6 \cdot 8 \\
2 \cdot 7 \\
0\end{array}$ & $\begin{array}{r}54 \\
28 \\
19 \\
20 \\
7 \\
8 \\
1\end{array}$ & $\begin{array}{r}41 \cdot 9 \\
21 \cdot 7 \\
14 \cdot 7 \\
15 \cdot 5 \\
5 \cdot 4 \\
6 \cdot 2 \\
0 \cdot 8\end{array}$ \\
\hline
\end{tabular}

values in $\mathbf{7 4}$ women in the study group are compared with that in 137 women in the control group who did not have trichomoniasis and who were not menstruating (controls only). The mean $\mathrm{pH}$ value of women in the study group was $5 \cdot 1$, which was similar to the mean of 5.2 for the healthy controls. The mean $\mathrm{pH}$ of women with trichomoniasis in both groups (5.6 and 5.8) was higher than that for women with no infection and no abnormal discharge $(4 \cdot 8$ and $5 \cdot 1$ ) (Table 3 ).

Of 27 women in the study group who had $N$. gonorrhoeae cultured from any site (Table 4$)$, the urethra gave a positive result in $88.9 \%$ and was the

Table 1 Rates of gonorrhoea, trichomoniasis, and yeast vaginal infection in 91 women after hysterectomy and in 264 healthy controls

\begin{tabular}{|c|c|c|c|c|c|c|}
\hline \multirow[b]{2}{*}{ Infection } & \multicolumn{2}{|c|}{ Study group $(n=91)$} & \multicolumn{2}{|c|}{ Control group $(n=264)$} & \multirow[b]{2}{*}{$z \operatorname{tes} t^{*}$} & \multirow[b]{2}{*}{ Probability } \\
\hline & No. & $\%$ of total & No. & $\%$ of total & & \\
\hline $\begin{array}{l}\text { Gonorrhoea } \\
\text { Trichomoniasis } \\
\text { Yeast }\end{array}$ & $\begin{array}{l}12 \\
17 \\
10\end{array}$ & $\begin{array}{l}13 \cdot 2 \\
18 \cdot 7 \\
11 \cdot 0\end{array}$ & $\begin{array}{l}34 \\
23 \\
19\end{array}$ & $\begin{array}{r}12 \cdot 9 \\
8 \cdot 7 \\
7 \cdot 2\end{array}$ & $\begin{array}{l}0 \cdot 0755 \\
2 \cdot 5937 \\
1 \cdot 1389\end{array}$ & $\begin{array}{l}\text { NS } \\
<0 \cdot 01 \\
\text { NS }\end{array}$ \\
\hline
\end{tabular}

* Critical ratio for the difference of proportions

NS = not significant 
Table 3 Comparison of vaginal pH in 61 women after hysterectomy, and in 99 healthy controls, some of whom had gonorrhoea, trichomoniasis, yeast infection, or no genital infection or abnormal discharge

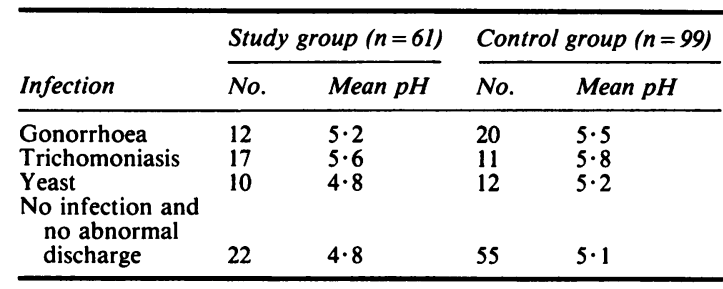

Table 4 Comparison of urethral and vaginal culture results in 27 women with anogenital gonorrhoea who had undergone hysterectomy

\begin{tabular}{|c|c|c|c|}
\hline \multicolumn{4}{|c|}{$\begin{array}{l}\text { Culture results for specimens } \\
\text { from }\end{array}$} \\
\hline Urethra & Vagina & No. & $\%$ of total \\
\hline+ & + & 13 & $48 \cdot 2$ \\
\hline+ & - & 11 & $40 \cdot 7$ \\
\hline - & + & 2 & $7 \cdot 4$ \\
\hline - & - & $1^{*}$ & $3 \cdot 7$ \\
\hline
\end{tabular}

only site giving a positive result in $40 \cdot 1 \%$ compared with the vagina, which gave a positive result in $55.6 \%$ and was the only site giving a positive result in $7 \cdot 4 \%$. The anal canal gave positive results in 11 $(40 \cdot 7 \%)$ and was the only site giving a positive result in one $(3 \cdot 7 \%)$.

\section{Discussion}

The uterine cervix is a secretory organ lined by mucus-producing columnar epithelial cells which serves as a conduit for endometrial secretions in one direction and spermatozoa and microbes in the other. Once genital contact with a pathogen has occurred, the cervix can affect both the rate of acquisition and the course of sexually transmitted genital infections.

Because $N$. gonorrhoeae is more frequently isolated from the endocervix than from the vagina (Dans and Judson, 1975), it has been argued that the endocervix is preferentially susceptible to gonorrhoea, possibly because of its columnar epithelial lining (Harkness, 1948). Brown and Lucas (1973) have even stated that 'the gonococcus is unable to penetrate normal stratified squamous epithelium and infection develops only on those surfaces lined with vulnerable columnar epithelium'. However, this opinion is contradicted by Evans's (1977) ultrastructural studies, which show primary squamous cell infection, and by clinical studies that document infections of the posterior pharynx
(Wiesner et al., 1973), the vagina and not the cervix in prepubescent girls (Harkness, 1948), the vagina in women after hysterectomy (Klaus et al., 1978), the urethra in women (Dans et al., 1975), and the anal canal in men and women (Dans et al., 1975).

Harkness (1948) has proposed that while the gonococcus may infect vaginal squamous epithelium it more avidly attacks columnar epithelium and hence the cervix with its abundance of this cell type. Evans (1977), however, was unable to demonstrate gonococci in or on cervical epithelium from 25 women with gonococcal cervicitis, finding it instead within and on stratified squamous epithelium of the portio vaginalis. Thus, the question of which cell type $N$. gonorrhoeae prefers remains unanswered.

Other factors which might be important to preferential endocervical survival of gonococci may be the qualitatively different microflora (Bartlett et $a l ., 1978$ ) and the higher $\mathrm{pH}$ of the cervical os. The usual $\mathrm{pH}$ value of secretions from the os is about $6 \cdot 8$ (Peeters et al., 1972) compared with 5.0 for vaginal secretions and 7.5 for the optimal growth of $N$. gonorrhoeae (Cohen, 1969). Cervical mucus itself may cyclically affect bacterial growth since it has been shown to be bactericidal for Proteus mirabilis (Enhorning et al., 1970). A recent study by Bartlett $e t$ al. (1978) comparing cervical and vaginal bacterial flora undermines much of this argument, for on the whole there were no quantitative differences in the flora of the two sites.

Furthermore, menstrual blood which passes through the uterine cervix is an excellent culture medium and could buffer vaginal $\mathrm{pH}$ into a range more conducive to the growth of $N$. gonorrhoeae. In reality this may not be important, as Dans and Judson (1975) have shown that endocervical culture positivity rates do not vary during the menstrual cycle.

Hysterectomy, by removing any predisposing effects of the endocervix to gonococci and by leaving behind primarily vaginal and urethral stratified squamous epithelium, should provide a model to resolve partly some of these controversies. We found no significant difference between the prevalence rates of genital gonorrhoea in women who had undergone hysterectomy and in matched healthy women. More importantly, the gonorrhoea attack rate in women exposed to gonorrhoea after hysterectomy was similar to that in healthy women exposed to gonorrhoea $(\mathrm{P}>0 \cdot 2)$. Thus, although the gonococcus has been assumed to favour cervical epithelium, it establishes infection quite well in its absence. Unfortunately, our study methods did not permit us to determine what, if any, contribution was made by infection of columnar epithelium within perivaginal and periurethral glands to the positive culture rate. 
For women with an intact uterus, the endocervix and anal canal have been the preferred sites from which to culture $N$. gonorrhoeae (Center for Disease Control, 1974). Similar guidelines for women who have undergone hysterectomy do not exist, but our study clearly indicates that urethral cultures are the most sensitive, detecting infection in $89 \%$ of 27 women with anogenital gonorrhoea after hysterectomy. This agrees, in general, with the recent report of Klaus et al. (1978), in which urethral, vaginal, and anal canal cultures gave a positive result in 100,41 , and $12 \%$ respectively of 17 women with anogenital gonorrhoea after hysterectomy.

Although we did not take urethral specimens for culture from our healthy women, previous studies have shown that after endocervical cultures vaginal rather than urethral cultures are the next most sensitive in detecting genital gonorrhoea. Thus, in 432 women with gonococci isolated at any anogenital site, Dans et al. (1975) found the endocervix to give a positive result in $96.1 \%$, the vagina in $84.9 \%$, and the urethra in $65 \%$. Lucas et al. (1967) obtained similar results in 1268 women with gonorrhoea; the endocervix gave a positive result in $86 \%$, the vagina in $79 \%$, and the urethra in $60 \%$. The greater sensitivity of a vaginal culture in women with an intact uterus is probably best explained by the discharging of viable gonococci from the cervix into the vagina.

As with the endocervix, the reasons for preferential colonisation of the urethra are not known but probably relate to the same, as yet poorly defined, combination of factors such as $\mathrm{pH}$, competing microflora, and cell type. To obtain the greatest sensitivity, we recommend that specimens from all three sites be cultured routinely.

In Gry's study (1964) of the prevalence of $T$. vaginalis in 387 women, the organism was cultured from cervical specimens in only $13 \cdot 1 \%$. It was never cultured from the cervix alone, and thus the presence of a cervix seems to have little effect on the prevalence of trichomoniasis. However, optimal growth of $T$. vaginalis occurs during menses (Banner, 1974). If this were due solely to the buffering action of menstrual blood, we would expect a lower prevalence of trichomoniasis in women after hysterectomy. Inexplicably, we found trichomoniasis to be significantly more common in women who had undergone hysterectomy than in healthy women $(18 \cdot 7 \%$ compared with $8 \cdot 7 \%, \mathrm{P}<0 \cdot 01)$. This may result from our methods of selection in that a significantly greater proportion of women who had undergone hysterectomy complained of vaginal discharge. The explanation does not lie in vaginal acidity, because with or without trichomoniasis, the mean $\mathrm{pH}$ value of women after hysterectomy did not significantly differ from that of the control women. It is not known whether the high $\mathrm{pH}$ associated with trichomoniasis is actually caused by the protozoon or instead helps to provide a favourable environment for infection (Cohen, 1969).

Candidosis is seldom sexually transmitted (Miles $e t$ al., 1977) but is nonetheless a common genital infection which could be influenced by the presence of the cervix. Rosenberg (1976) found that only $44 \%$ of women with the 'typical symptoms complex' of candidosis had positive results when cultures were performed throughout the cycle, while $85 \%$ had positive results when cultures were performed within four days of the onset of menstruation. If a change in premenstrual cervical mucus was responsible, the prevalence of yeast infections in women after hysterectomy would be lower than in controls. However, we found yeasts on $\mathrm{KOH}$ preparations in $11 \%$ of women who had undergone hysterectomy and in $7 \cdot 2 \%$ of control women $(P>0 \cdot 2)$. As with trichomoniasis, the slightly higher prevalence of yeast infections in women after hysterectomy may be due to the higher percentage of these women who were symptomatic.

The automated clinic data system is supported by a contract from the Operations Research Branch, Venereal Disease Control Division, Center for Disease Control, Atlanta, Georgia. The contributions of $\mathrm{Mr}$ Arnold Maltz, in data programming, of Dr Richard Rothenberg, in statistical analysis, of Ms Fern Lemaster, for secretarial assistance, and of the staff of the Denver Metro Health Clinic are gratefully acknowledged.

\section{References}

Banner, E. (1974). Vaginitis. Medical Clinics of North America, 58, 759-768.

Bartlett, J. and Moon, N. (1978). Cervical and vaginal bacterial flora: Ecological niches in the female lower genital tract. American Journal of Obstetrics and Gynecology, 130, 658-661.

Brown, W. and Lucas, J. (1973). Tice's Practice of Medicine, volume III, chapter 19, p. 1, Harper and Row Inc: Hagerstown, Maryland.

Center for Disease Control (1974). Criteria and Techniques for the Diagnosis of Gonorrhoea. US Public Health Services publication. Washington DC

Cohen, L. (1969). Influence of $\mathrm{pH}$ on vaginal discharges. British Journal of Venereal Disease, 45, 241-246.

Dans, P. and Judson, F. (1975). The establishment of a venereal disease clinic: II. An appraisal of current diagnostic methods in uncomplicated urogenital and rectal gonorrhoea. Journal of the American Venereal Disease Association, 1, 107-112.

Enhorning, G., Huldt, L., and Melen, B. (1970). Ability of cervical mucus to act as a barrier against bacteria. American Journal of Obstetrics and Gynecology, 108, 532-537.

Evans, B. (1977). Ultrastructural study of cervical gonorrhoea. Journal of Infectious Diseases, 136, 248-255.

Grys, E. (1964). Examination of women for Trichomonas vaginalis in rural foci of infection. Waid Parazytal, 10, 122-129.

Harkness, A. (1948). The pathology of gonorrhoea. British Journal of Venereal Diseases, 24, 137-141. 
Klaus, B., Chandler, J., and Dans, P. (1978). Gonorrhoea detection in posthysterectomy patients. Journal of the American Medical Association, 240, 1360-1361.

Lucas, J., Price, E., Thayer, J., and Schroeter, A. (1967). Diagnosis and treatment of gonorrhoea in the female. New England Journal of Medicine, 26, 1454-1459.

Miles, M., Olsen, L., and Rogers, A. (1977). Recurrent vaginal candidiasis. Journal of the American Medical Association, 238 , 1836-1837.

Peeters, F., Snauwaert, R., and Segers, J. (1972). Observations on candidal vaginitis. American Journal of Obstetrics and Gynecology, 112, 80-96.
Rosenberg, M. (1976). Vaginal candidiasis: Its diagnosis and relation to urinary infection. Southern Medical Journal, 69, 1347-1348.

Rothenberg, R. and Judson, F. (1979). The Venereal Disease Strategic Planning System: record keeping in a clinic for sexually transmitted dieseases. Sexually Transmitted Diseases, 6, 1-4.

Wiesner, P., Tronca, E., Bonin, P., Pedersen, A., and Holmes, K. (1973). Clinical spectrum of pharyngeal gonococcal infection. New England Journal of Medicine, 288, 181-185. 